\title{
Comparison of culture, confocal microscopy and PCR in routine hospital use for microbial keratitis diagnosis
}

\author{
Jeremy J. Hoffman $\mathbb{D}^{1,2,3^{凶}}$, John K. G. Dart ${ }^{1,3}$, Surjo K. De ${ }^{1,4}$, Nicole Carnt ${ }^{1}{ }^{1}$, Georgia Cleary ${ }^{1}$ and Scott Hau ${ }^{1}$ \\ (c) The Author(s) 2021
}

\begin{abstract}
AIMS: To evaluate the sensitivity and specificity of polymerase chain reaction (PCR), in vivo confocal microscopy (IVCM) and culture for microbial keratitis (MK) diagnosis.

METHODS: Retrospective review of PCR, IVCM and culture results for MK diagnosis at Moorfields Eye Hospital between August 2013 and December 2014.

RESULTS: PCR results were available for 259 MK patients with concurrent culture for 203/259 and IVCM for 149/259. Sensitivities and specificities with 95\% confidence intervals [95\% Cl] were calculated for Acanthamoeba keratitis (AK) and fungal keratitis (FK), by comparison with culture, for both IVCM and PCR. For AK, FK and bacterial keratitis (BK) sensitivities were calculated, for each diagnostic method, by comparison with a composite reference standard (a positive result for one or more of culture, PCR or IVCM having a specificity of $100 \%$ by definition). For the latter, sensitivities with [95\% CI] were: for AK, IVCM 77.1\% [62.7-88.0\%], PCR 63.3\% [48.3-76.6\%], culture 35.6 [21.9-51.2]; for FK, IVCM 81.8\% [48.2-97.7\%], PCR 30.8\% [9.09-61.4\%], culture 41.7\% [15.2-72.3\%]; for BK, PCR 25.0\% [14.7-37.9\%], culture 95.6\% [87.6-99.1\%].

CONCLUSION: IVCM was the most sensitive technique for AK and FK diagnosis but culture remains our gold standard for BK. These findings reflect results to be expected from service providers to UK ophthalmology units and demonstrates the need at our centre for ongoing diagnostic result audit leading to the potential to improve PCR diagnosis. Both FK and AK are now common in the UK; ophthalmology units need to have all these techniques available to optimise their MK management.
\end{abstract}

Eye (2022) 36:2172-2178; https://doi.org/10.1038/s41433-021-01812-7

\section{INTRODUCTION}

Microbial keratitis (MK) can be caused by a diverse range of microorganisms; accurate, early diagnosis of the causative organism is crucial to the choice of an appropriate antimicrobial treatment that is required for a good outcome. Empirical treatment for MK is widely used, without investigations, despite the overlap of clinical signs for the major groups of causative organisms; bacteria, Acanthamoeba and fungi, for which treatments are quite different.

The traditional 'gold standard' for diagnosing MK is microbiological diagnosis with microscopy and culture. However, the sensitivity of culture is poor with culture-positive rates ranging from 32.6 to $79.4 \%$ [1], increasing with the size of the ulcer [2]. Microscopy of stained corneal tissue (corneal biopsy and/or smear) achieves rates of 27.3 to $61.6 \%$ depending on the stain used and organism being identified [1]. Alternative tools, including polymerase chain reaction (PCR) and in vivo confocal microscopy (IVCM), have been developed to aid in the diagnosis of MK and are used in combination with culture and microscopy in some tertiary referral centres to help improve diagnostic precision in terms of sensitivity and specificity.

Although a number of studies have investigated the sensitivity and specificity of culture, PCR and IVCM for either fungal keratitis (FK) or Acanthamoeba keratitis (AK) [1, 3-8], these have often focused on one diagnostic modality for comparison, or have been specific to either bacterial keratitis (BK), FK or AK. The purpose of this study was to compare the sensitivity and specificity of IVCM, PCR, and corneal cultures, in routine clinical practice at our tertiary referral centre and using our external pathology service providers, for a consecutive cohort of patients presenting to our centre with MK of any cause. These patients include both primary and tertiary referrals.

\section{SUBJECTS AND METHODS \\ Participants}

This study was approved by Moorfields Eye Hospital (MEH) Clinical Audit and Assessment Committee (Ref: CA14/CED/38) and adhered to the tenets of the Declaration of Helsinki as a retrospective, observational case note review of patients who attended MEH with MK between 14th August 2013-6th December 2014. All three investigations (culture, PCR and IVCM) were being performed routinely at this point, and this study was performed to audit their results a few months after PCR was introduced. The inclusion criteria were patients with MK during this period who had undergone PCR.

\section{Culture and PCR}

Patients with MK attending MEH are assessed using an in-house protocol available on the Microguide App (http://www.microguide.eu/services/

\footnotetext{
${ }^{1}$ Moorfields Eye Hospital NHS Foundation Trust, London, UK. ${ }^{2}$ International Centre for Eye Health, London School of Hygiene and Tropical Medicine, London, UK. ${ }^{3}$ National

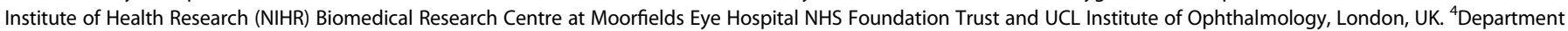
of Microbiology, University College London Hospitals NHS Foundation Trust, London, UK. ${ }^{凶}$ email: j.hoffman@ucl.ac.uk
} 
mobile/), known as the Moorfields Emergency Guideline App. PCR, culture and IVCM are carried out on presentation if there is severe disease (a large ulcer or abscess with or without a hypopyon or ring infiltrate) or, for early disease, having features suggestive of non-bacterial keratitis or 'atypical keratitis'. These investigations are also performed when keratitis cases are unresponsive to therapy.

PCR samples were collected by corneal swabs and sent to an external laboratory for analysis (Micropathology Ltd, Coventry, UK). The assays requested included 16S rRNA PCR (pan-bacterial), 18S rRNA PCR (panfungal), Acanthamoeba species-specific PCR, Aspergillus genus DNA PCR, Candida albicans DNA PCR, cytomegalovirus DNA PCR, Epstein-Barr virus DNA PCR, Group A Streptococcus DNA PCR, Group B Streptococcus DNA, Herpes Simplex Virus DNA PCR, Mycobacterium genus DNA PCR, varicella zoster virus DNA PCR and Staphylococcus DNA PCR. Corneal scrapings were collected in duplicate for microscopy and culture using fresh 21-gauge needles after anaesthetizing the eye with topical $0.5 \%$ proxymetacaine hydrochloride (Bausch \& Lomb, U.K. Ltd). These were inoculated directly onto culture media (blood agar, Sabouraud's dextrose agar for fungi, brain-heart-infusion broth and Escherichia coli-seeded non-nutrient agar subsequently seeded with Escherichia coli for Acanthamoeba) and sent for processing and reporting by an external service (The Doctors Laboratory Ltd, London, UK).

\section{In vivo confocal microscopy}

IVCM was performed by trained experienced operators using the HRT II/ RCM confocal microscope (Heidelberg Engineering, Dossenheim, Germany) using a previously described standard operating procedure [8]. Findings indicative of Acanthamoeba or FK were as previously reported and classified accordingly $[4,7,9]$. Bacteria, with the exception of Nocardia spp., are too small to be detected by IVCM [10]. All the images were reviewed and classified into the various type of keratitis, in a masked fashion, by one experienced observer $(\mathrm{SH})$.

\section{Disease definition and analysis}

Disease definition was based on a positive diagnosis using culture, PCR or IVCM. To ensure we were assessing the sensitivity and specificity of investigations, the clinical findings and patient response to antimicrobial treatment were not used in the analysis or for disease definition. The diagnostic capability of the various tests was evaluated by determining: (1) the sensitivity and specificity of PCR and IVCM in comparison to culture and (2) if a composite reference standard, in which a positive result from one or more of the techniques confirms the diagnosis, is superior to the historical 'gold' standard of culture. The reference standards for comparison are defined as follows:

- Culture: based on a positive result obtained from culture only.

- PCR: positive result from PCR only.

- IVCM: organism identified on imaging.

- Composite MK diagnosis: a positive result for at least 1 of culture, PCR or IVCM [8].

\section{Statistical analysis}

For one analysis we have compared PCR, IVCM and culture for Acanthamoeba and filamentous fungus using each as a reference standard for comparison with the others. For the other analysis we have compared the results for Culture, PCR and IVCM (the latter not for bacteria) with the composite reference standard.

Data were collated in Microsoft Excel 2019 and analysed using STATA 15. We calculated sensitivity and specificity values, including exact binomial confidence intervals, for each diagnostic modality compared to the various reference standards.

\section{RESULTS}

A total of 259 patients with unilateral MK [52.9\% female; mean age 52.7 years (SD 20.8; range 6-108)] had PCR samples analysed.

Table 1 describes the organisms detected, categorised by microbial group, for 259 patients with a clinical diagnosis of microbial keratitis using culture and/or PCR and/or IVCM. No organisms were detected in 128/259 (49.4\%) of cases by any technique. PCR was used in all 259 cases, 203/259 had PCR and culture, and 149/259 had PCR and IVCM. However, although the numbers for culture and IVCM were fewer than the totals tested by PCR, most cases that were positive using a composite diagnosis (all techniques combined) had had all the tests performed-the few exceptions are mentioned in the text describing results for individual groups of organisms below. For all tests combined (any test positive) $131 / 259$ subjects $(50.6 \%)$ had positive results. The positive results by detection method for each category of organism are also shown in Table 1 and are summarised in Fig. 1, in which the Venn diagrams show the numbers positive by one or more tests.

\section{Bacterial keratitis}

There were 54 monomicrobial bacterial keratitis cases, of which one did not undergo culture but in which PCR was positive (Klebsiella spp.). IVCM was not performed routinely for BK as the resolution is inadequate to visualise bacteria other than Nocardia $\mathrm{sp}$. or to visualise infectious crystalline keratopathy (ICK). In our cohort, there were three cases of ICK imaged by IVCM; two were confirmed as bacterial on culture and one as Acanthamoeba by PCR. There were no cases of Nocardia in our cohort. Bacteria were also involved in 16 cases of polymicrobial keratitis (3 with filamentous fungus and 12 with Acanthamoeba) totalling 70 cases in which bacteria were a cause. Table 1 and Fig. 1A show that culture was positive in 65/70 (92.8\%) of BK cases compared to PCR in $15 / 70(21.4 \%)$. Figure $1 \mathrm{~A}$ shows culture negative $\mathrm{BK}$ cases and the one case that did not undergo culture were diagnosed by PCR alone in only 5/70 (7.1\%) cases. Supplementary Table 1 lists the bacterial organisms identified by culture and which of these were also detected by PCR.

\section{Acanthamoeba keratitis}

Of the 50 cases of AK there were 38 monomicrobial and 12 polymicrobial in combination with BK. Culture was not performed for five cases, PCR not analysed for one case and IVCM not carried out for two cases. Table 1 and Fig. 1B show that IVCM was positive in $37 / 50(74 \%)$ compared to PCR in $31 / 50(62 \%)$ and culture in $16 /$ 50 (32\%). Figure 1B shows that IVCM negative cases were diagnosed by PCR or culture, either alone or in combination, in $9 / 50(18 \%)$ cases.

\section{Filamentous fungal keratitis and yeasts}

Of the 14 cases of filamentous FK and 1 case of yeast keratitis, culture was not performed for one case of filamentous FK, PCR was not analysed for one and IVCM not carried out for one. The case of yeast FK (Candida dubliniensis by sequencing) was budding and identified by all three methods. Table 1 and Fig. $1 \mathrm{C}$ show that IVCM was positive in $10 / 15(66.7 \%)$ identifying the highest proportion of cases compared to PCR in 5/15 (33.3\%) and culture in $6 / 15(40 \%)$. Figure $1 C$ shows that IVCM negative cases were diagnosed with either PCR or culture in 5/15 (33.3\%) of cases. Supplementary Table 2 lists the fungal organisms identified by culture and which of these were also detected by PCR and / or IVCM.

\section{Polymicrobial (mixed) infection}

For mixed Acanthamoeba and bacterial infections, all 12 cases were culture positive for bacteria versus $1 / 9$ tested by PCR. For polymicrobial fungal and bacterial infections, $3 / 3$ were positive for bacteria on culture versus $1 / 3$ for PCR. IVCM and PCR were more often positive than culture for the fungal and Acanthamoeba components of these polymicrobial cases. All the mixed bacterial/fungal infections were caused by filamentous fungi as opposed to yeasts.

\section{Viral keratitis}

Of the 12 cases of viral keratitis, PCR detected herpes simplex virus in ten cases and varicella zoster virus (VZV) in two cases. This is the 
Table 1. Organism(s) detected in 259 keratitis patients categorised by group of organism and method of detection for culture, polymerase chain reaction (PCR) and in vivo confocal microscopy (IVCM) both separately and for the composite diagnosis using all methods combined.

\section{Diagnostic methods}

All methods combined
Culture \pm PCR \pm IVCM
(Composite microbial keratitis
diagnosis)

\begin{tabular}{|c|c|c|c|c|c|c|c|c|c|}
\hline \multicolumn{2}{|c|}{ Numbers of subjects tested } & $n$ & (\%) & $n$ & (\%) & $n$ & (\%) & $n$ & (\%) \\
\hline \multicolumn{2}{|l|}{ Total number } & 259 & $(100)$ & 203 & $(100)$ & 259 & $(100)$ & 149 & $(100)$ \\
\hline \multicolumn{2}{|l|}{ For Bacteria } & & & 203 & $(100)$ & 189 & $(73.0)$ & $\mathrm{n} / \mathrm{a}$ & \\
\hline \multicolumn{2}{|c|}{ For Acanthamoeba } & & & 203 & $(100)$ & 175 & $(67.6)$ & 149 & $(100)$ \\
\hline \multicolumn{2}{|c|}{ For Filamentary fungus } & & & 203 & $(100)$ & 180 & $(69.4)$ & 149 & $(100)$ \\
\hline \multicolumn{2}{|c|}{ Total negative } & $128 / 259$ & $(49.4)$ & 116 & $(57.1)$ & 195 & $(75.3)$ & 102 & $(68.5)$ \\
\hline \multicolumn{2}{|c|}{ Negative for Bacteria } & & & $135 / 203$ & $(66.5)$ & $129 / 195$ & $(66.2)$ & $\mathrm{n} / \mathrm{a}$ & \\
\hline \multicolumn{2}{|c|}{ Negative for Filamentary fungus } & & & $190 / 203$ & $(93.6)$ & $166 / 195$ & $(85.1)$ & 137 & (91.9) \\
\hline \multicolumn{2}{|c|}{ Total Positive (yield) } & $131 / 259$ & $(50.6)$ & $87 / 203$ & $(42.9)$ & 64 & $(24.7)$ & 47 & $(31.5 \%)$ \\
\hline \multirow{2}{*}{\multicolumn{4}{|c|}{$\begin{array}{l}\text { Positive results (yield) by } \\
\text { organism groups }\end{array}$}} & \multicolumn{6}{|c|}{$\begin{array}{l}\text { Bacteria, fungi and Acanthamoeba were identified by more than one } \\
\text { method; the numbers identified by multiple methods are described in } \\
\text { Fig. } 1\end{array}$} \\
\hline & & & & \multicolumn{2}{|c|}{ Culture positive } & \multicolumn{2}{|c|}{ PCR positive } & \multicolumn{2}{|c|}{ IVCM positive } \\
\hline \multicolumn{2}{|c|}{ Acanthamoeba } & 38 & (14.7) & $15 / 33$ & $(45.5)$ & $24 / 37$ & $(64.9)$ & $26 / 36$ & $(72.2)$ \\
\hline \multicolumn{2}{|c|}{ Herpes simplex virus } & 10 & (3.9) & $\mathrm{n} / \mathrm{a}$ & & $10 / 10$ & $(100)$ & $\mathrm{n} / \mathrm{a}$ & \\
\hline \multicolumn{2}{|c|}{ Varicella zoster virus } & 2 & $(0.8)$ & $n / a$ & & $2 / 2$ & $(100)$ & $n / a$ & \\
\hline \multirow[t]{4}{*}{ Polymicrobial } & Bacteria WITH & 4 & (1.5) & $3 / 3$ & (100) & $1 / 2$ & (50) & $n / a$ & \\
\hline & Filamentary Fungal $^{a}$ & & & $1 / 3$ & (33.3) & $2 / 2$ & $(100)$ & $2 / 2$ & $(100)$ \\
\hline & Bacteria WITH & 12 & (4.6) & $12 / 12$ & $(100)$ & $1 / 9$ & $(11.1)$ & $\mathrm{n} / \mathrm{a}$ & \\
\hline & Acanthamoeba & & & $1 / 12$ & $(8.3)$ & $7 / 9$ & (77.8) & $11 / 12$ & (91.7) \\
\hline
\end{tabular}

${ }^{a}$ All mixed bacterial/fungus infections were filamentary; there were no mixed bacterial/yeast infections in the course of this study.

only diagnostic test available at our facility for viral keratitis, only performed if there is uncertainty regarding clinical diagnosis, and so was not used to generate sensitivity and specificity data.

\section{Sensitivity and specificity}

Table 2 shows the summary statistics of the sensitivity and specificity values for PCR, IVCM, and cultures for both FK and AK, compared to the different reference standards. When compared to the 'gold' reference standard of culture, the sensitivity of PCR was higher for AK vs FK whereas IVCM was higher for FK vs AK, although the specificities were similar for both. The sensitivities of PCR and IVCM were similar for AK, whereas IVCM performed substantially better vs PCR for FK. The positive and negative test results for calculating these indices for this table are included in Supplementary Table 3.

Table 3 gives the sensitivity and specificity of PCR, IVCM and culture for AK and FK, as compared to the composite diagnosis reference standard. For AK, the highest sensitivity was with IVCM and lowest with culture. For FK, the highest sensitivity was also IVCM, however culture performed better than PCR. For bacteria, culture was substantially more sensitive than PCR. The specificities for all three diagnostic modalities were $100 \%$ when compared to the composite diagnosis reference standard because the composite reference by definition means that there will be no false positive results, as all positive results are included within the composite diagnosis category.

\section{Culture alone \\ PCR alone \\ IVCM alone}

\section{(1)}


A Bacterial keratitis $(n=70)$

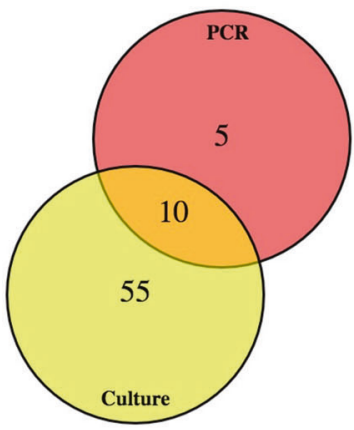

Culture positive $n=65$

PCR positive $\quad \mathrm{n}=15$

IVCM not done
B Acanthamoeba keratitis $(\mathrm{n}=50)$

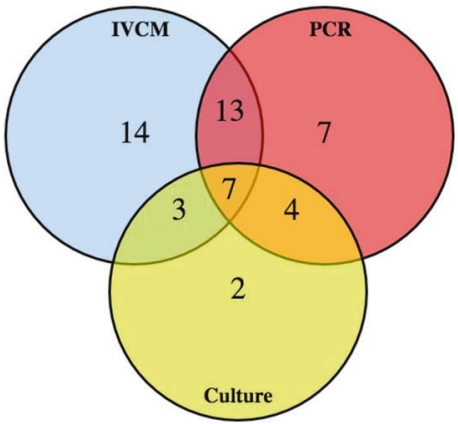

Culture positive $\mathrm{n}=16$

PCR positive $\quad \mathrm{n}=31$

IVCM positive $n=37$
C Fungal keratitis $(\mathrm{n}=15)$

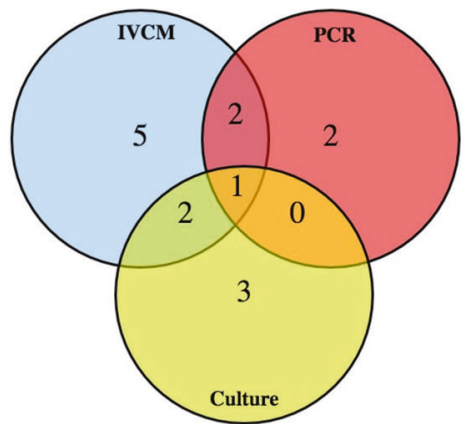

Culture positive $n=6$

PCR positive $\quad \mathrm{n}=5$

IVCM positive $\mathrm{n}=10$

Fig. 1 Venn diagram showing the number of cases that were positive for bacteria, Acanthamoeba and fungal keratitis using culture, polymerase chain reaction (PCR) and in vivo confocal microscopy (IVCM). Cases that were positive for more than one test are given within the overlapping areas. A Investigations that were positive for the 70 cases of bacterial keratitis including mixed infections. Note IVCM not included for bacterial keratitis; B Investigations that were positive for the 50 cases with Acanthamoeba keratitis including mixed infections; C Investigations that were positive for the 15 cases with fungal keratitis including mixed infections and the one case of Candida dubliensis infection, which was positive in all three investigations.

Table 2. Sensitivity and specificity values with $95 \%$ confidence intervals (brackets) of culture, polymerase chain reaction and in vivo confocal microscopy compared to different reference standards for Acanthamoeba and filamentary fungus. Full details for calculating the sensitivity and specificity for each modality are given in online Supplementary Table 1.

\begin{tabular}{|c|c|c|c|c|c|c|}
\hline \multirow[t]{3}{*}{ Diagnostic method } & \multicolumn{6}{|c|}{ Reference standard } \\
\hline & \multicolumn{3}{|l|}{ Acanthamoeba } & \multicolumn{3}{|c|}{ Filamentary fungus } \\
\hline & Culture & PCR & IVCM & Culture & PCR & IVCM \\
\hline Sensitivity \% & Referent & $40.7(22.4-61.2)$ & $29.4(15.1-47.5)$ & Referent & $25.0(0.63-80.6)$ & $37.5(8.52-75.5)$ \\
\hline Specificity \% & Referent & $96.7(91.8-99.1)$ & $95.7(89.5-98.8)$ & Referent & $97.4(93.4-99.3)$ & 98.3 (94.1-99.8) \\
\hline Specificity \% & $88.1(81.3-93.0)$ & Referent & $83.5(74.6-90.3)$ & $98.0(94.3-99.6)$ & Referent & $100(96.3-100)$ \\
\hline \multicolumn{7}{|l|}{ IVCM } \\
\hline Sensitivity \% & 71.4 (41.9-91.6) & $69.0(49.2-84.7)$ & Referent & $60.0(14.7-94.7)$ & $100(29.2-100)$ & Referent \\
\hline Specificity \% & $78.9(70.3-86.0)$ & $83.5(74.6-90.3)$ & Referent & $95.9(90.8-98.7)$ & $93.4(86.9-97.3)$ & Referent \\
\hline
\end{tabular}

$P C R$ polymerase chain reaction, IVCM in vivo confocal microscopy.

BK accounted for the highest proportion of cases of MK $(70 / 131$ cases, 53.4\%), and FK for the lowest in this study, in keeping with other studies at temperate latitudes [11-15]. Acanthamoeba keratitis was identified in 50/131 cases (38.2\%) which is higher than reported in previous studies [11-13]. This is likely to be due to three reasons: the fact that a clinical diagnosis was not used in the analysis which will have resulted in failure to identify probable BK in many small bacterial keratitis lesions which are more often culture negative [16], that Moorfields is a tertiary referral centre $[17,18]$, and that the data for this study was collected during an outbreak of AK in the South East of the UK [17].

Limitations of this study are several. To ensure we were evaluating the yield, sensitivity and specificity of these techniques, the clinical diagnosis (clinical findings and response to antimicrobial treatment) was not used as a comparator and it is not possible to be certain of the cause of keratitis in the 128/259 (49.4\%) of patients in whom all diagnostic tests were negative. The low total yield $(50.6 \%)$ identified in our study may itself bias the results as we can be unsure of the diagnostic accuracy of the patients who tested negative to all three tests but yet had a clinical diagnosis of MK. Microscopy results were not included as these were not accessible. Our masked observer is very experienced in analysing IVCM keratitis images making extrapolation of these results to units having less experienced IVCM operators uncertain. Not every case had every investigation performed, although most cases with a positive diagnosis by one test also had the other tests performed (Table 1). As a result, the numbers of patients within the composite diagnosis reference group to whom the different diagnostic investigations compared to in Table 3 differ between organism group and type of investigation, which may result in some bias. The number of FK cases in this series was low, resulting in wide confidence intervals. Finally, the processing and reporting of the cultures and material provided for PCR was carried out by external providers, using assays which may have had differing performance characteristics compared to those used in studies carried out in research laboratories using custom primers [19], or 
Table 3. Sensitivity and specificity values for detecting Acanthamoeba, filamentary fungus and bacteria using PCR, IVCM and culture compared to a composite diagnosis reference standard. The composite diagnosis reference standard is where an individual tests positive for an organism group (Acanthamoeba, bacteria or fungus) in one or more of the three diagnostic investigations. The sensitivity/specificity values are shown on the column to the right and the number of positive and negative test results, for the organism in question, are shown on the left.

\begin{tabular}{|c|c|c|c|c|c|}
\hline \multirow[t]{2}{*}{ Diagnostic Method $^{\mathrm{a}}$} & \multicolumn{2}{|c|}{ Composite Diagnosis Reference Standard } & \multirow[t]{2}{*}{ Totals $^{\mathbf{b}}$} & \multirow[t]{2}{*}{ Indices } & \multirow[t]{2}{*}{ Value $(\% \mathrm{Cl})$} \\
\hline & Positive & Negative & & & \\
\hline \multicolumn{6}{|c|}{ Acanthamoeba ( $n=50$ detected including both mono and polymicrobial) } \\
\hline Positive & 31 & 0 & 31 & Sensitivity \% & $63.3(8.3-76.6)$ \\
\hline Negative & 18 & 126 & 144 & Specificity \% & $100(97.1-100)$ \\
\hline Total & 49 & 126 & 175 & & \\
\hline Positive & 37 & 0 & 37 & Sensitivity \% & $77.1(62.7-88.0)$ \\
\hline Negative & 11 & 101 & 112 & Specificity \% & $100(96.4-100)$ \\
\hline Total & 48 & 101 & 149 & & \\
\hline \multicolumn{6}{|l|}{ Culture } \\
\hline Positive & 16 & 0 & 16 & Sensitivity \% & $35.6(21.9-51.2)$ \\
\hline \multicolumn{6}{|l|}{ PCR } \\
\hline Positive & 4 & 0 & 4 & Sensitivity \% & $30.8(9.09-61.4)$ \\
\hline Negative & 9 & 167 & 176 & Specificity \% & $100(97.8-100)$ \\
\hline Total & 13 & 167 & 180 & & \\
\hline \multicolumn{6}{|l|}{ IVCM } \\
\hline Positive & 9 & 0 & 9 & Sensitivity \% & $81.8(48.2-97.7)$ \\
\hline Negative & 2 & 138 & 140 & Specificity \% & $100(97.4-100)$ \\
\hline Total & 11 & 138 & 149 & & \\
\hline \multicolumn{6}{|l|}{ Culture } \\
\hline Positive & 5 & 0 & 5 & Sensitivity \% & $41.7(15.2-72.3)$ \\
\hline \multicolumn{6}{|c|}{ PCR } \\
\hline \multicolumn{6}{|l|}{ Culture } \\
\hline Positive & 65 & 0 & 65 & Sensitivity \% & $95.6(87.6-99.1)$ \\
\hline Negative & 3 & 135 & 138 & Specificity \% & $100(97.3-100)$ \\
\hline Total & 68 & 135 & 203 & & \\
\hline
\end{tabular}

$P C R$ polymerase chain reaction, IVCM in vivo confocal microscopy; $C l$ confidence interval.

aIVCM was not performed for cases of bacterial keratitis;

${ }^{b}$ The total number of individuals in the composite diagnosis reference standard differs for each organism group and investigation as not every individual had all three investigations performed. When comparing the tests for each organism group (bacteria, fungus or amoeba) to the composite reference standard, only the total number of patients who had the particular test in question being performed are included. Please refer to Table 1 for the number of diagnostic tests performed for each organism group in question.

techniques [20]. On the other hand, dedicated research microbiology laboratories are rarely available for clinical use which relies on service providers. As a result, the findings from this study represent what most ophthalmic units in the UK and elsewhere might expect from their external providers of microbiology facilities.

The strengths of this study include the use of a single experienced masked assessor for interpreting the IVCM images, with all imaging performed following the same standard operating procedure; the large sample size of our cohort; the use of a composite diagnosis for technique comparison and the pragmatic evaluation of clinical diagnostic services.

The overall yield of positive cultures of $87 / 203(42.9 \%)$ is consistent with findings from the UK, where a large series of MK including very small ulcers found that most cases of MK were caused by bacteria and found the culture positive rate was as low 
as $1379 / 4229$ (33\%) [21]. This is as opposed to the rates for cultures from large ulcers in India, where FK is more common than BK and culture positive rates are generally higher, ranging from $51.9 \%(56 / 108)$ for ulcers $\geq 1.0 \mathrm{~mm}$ [22], up to as high as $76 \%$ (182/ 239) for ulcers $\geq 3.00 \mathrm{~mm}$.

Supplementary Table 4 is a summary of studies comparing PCR, IVCM and culture/smear yields, sensitivity and specificity in microbial keratitis. For PCR the diagnosis rates vary depending on the reference standard used, the PCR primers, the organisms, the severity of disease, whether or not the patients have been treated with antimicrobials before the samples are taken, and the unquantifiable factors of the quality of the diagnostic facilities and the sample. The principal difference in sensitivity, specificity and yield, by comparison with the studies summarised here is the relatively low sensitivities in our study for fungal and bacterial diagnosis using PCR. PCR for bacterial keratitis has been reported as more sensitive than culture in a similar study to ours [23], but from a population having a higher positive diagnostic rate and one that includes smear microscopy as part of the composite reference (likely reducing the calculated sensitivity of culture), whilst another has shown similar PCR positive rates to culture positive rates [24]. In contrast, our study found bacterial PCR sensitivity of $25 \%(\mathrm{Cl} 14.7-37.9 \%)$ versus culture of $95.6 \%(\mathrm{Cl}$ 87.6-99.1\%). The higher rates of PCR sensitivity for bacterial keratitis reported elsewhere may have resulted from PCR false positives due to the detection of non-pathogenic bacteria as shown by Kim et al. [22], or better culture in combination with less effective PCR in our laboratories. Broad range 16S PCR has a limit of detection of between $10^{3}$ and $10^{4}$ colony forming units per millilitre [25], and our poor PCR sensitivity may result from samples being sent from less keratitis with less severe disease at presentation and/or that the samples we send have less than the optimal amount of material available for PCR. PCR for fungal keratitis has also been shown by most studies to be more often positive and more sensitive than culture [20, 23, 26, 27], with sensitivities between $70 \%$ and $93 \%$ compared to that of culture of $43 \%$ to $57 \%$; one study showed approximately the same yield using both techniques [28]. These findings compare to ours with a sensitivity for fungal PCR of $30.8 \%(\mathrm{Cl} 9.1-61.4 \%)$ versus culture of 41.7\% (Cl 15.2-17.3\%); the low sensitivity of PCR again being potentially due to early disease at presentation or poor sampling, as well as a poor choice of primers. Analysis of PCR for Acanthamoeba keratitis compared to culture has given variable results in different studies. Two studies show PCR, depending on the PCR primer used, to be more sensitive than culture with sensitivities ranging from $65 \%$ to $95 \%$ versus culture at $73.7 \%$ [29], or with a higher yield of $53 \%$ to $73 \%$ versus culture of $55 \%$ whereas two others with high rates of culture or smears positive (70-80\%) show a similar yield from PCR $[30,31]$. Our study shows comparable findings with sensitivity of $63 \%$ for Acanthamoeba PCR compared to $35.6 \%$ for culture.

IVCM diagnostic sensitivity depends, as for PCR, on the reference standard used which has been culture and/or microscopy in three studies $[3,5,7]$, these, with the addition of some clinically diagnosed cases in a fourth [32], and Acanthamoeba PCR in a fifth [33]. Two studies showed sensitivities of $80-90 \%$ with specificities of $80-90 \%$ for both AK and FK diagnosis [5, 7], similar to those for AK diagnosis in a third study [32]. However, a further study comparing IVCM diagnosis for Acanthamoeba, filamentous fungus, Nocardia and Microsporidia between five different observers found very variable results depending on the experience of the observer; the most experienced having a sensitivity of $55.8 \%$ and specificity of $84.2 \%$ [3]. Our findings in this study, using an experienced observer, are similar to these published studies having sensitivities [specificities] for Acanthamoeba and filamentous fungus respectively of $77.1 \%$ [100\%] and $81.8 \%$ [100\%]. The IVCM criteria used for AK diagnosis may also affect the sensitivity and specificity varying with the criteria used for diagnosis; those giving the highest sensitivity of $73.9 \%$ had the lowest specificity of $48.2 \%$ and those with the highest specificity of $98.2 \%$ having the lowest sensitivity of $15.2 \%$ [33]. However, sensitivity and specificity are not always of equal clinical importance; sensitivity may be deemed to be more useful than specificity in this case in order not to miss atypical organisms. This should be considered when interpreting the results of our study.

Our study shows what may be achieved for the diagnosis of MK using general (non-ophthalmic) culture and PCR service providers that are available to UK hospitals.

These findings may be useful to others as a benchmark for what can be expected for MK diagnosis in the UK and can be used to help improve the sensitivity of diagnostic tests for MK. Lastly, this study demonstrates that all three techniques are needed to optimise MK diagnosis and, given the increasing incidence of fungal and Acanthamoeba keratitis in the UK [21, 34], shows that these facilities should be made available to all UK ophthalmic units.

\section{Summary table}

What was known before

- The traditional "gold standard" for diagnosing MK is microbiological diagnosis with microscopy and culture.

- In vivo confocal microscopy and Polymerase Chain Reaction (PCR) are potentially useful adjunctive tools to diagnosis.

- The purpose of this study was to compare the sensitivity and specificity of IVCM, PCR, and corneal cultures, in routine clinical practice.

What this study adds

- In vivo confocal microscopy was the most accurate tool for diagnosing Acanthamoeba and fungal keratitis compared to PCR and culture.

- PCR was more sensitive for Acanthamoeba than fungus.

- Culture performed best for bacterial keratitis.

- Ophthalmology units in the UK need to have all these techniques available to optimise their MK management.

\section{REFERENCES}

1. Ung L, Bispo PJM, Shanbhag SS, Gilmore MS, Chodosh J. The persistent dilemma of microbial keratitis: Global burden, diagnosis, and antimicrobial resistance. Surv Ophthalmol. 2019;64:255-71.

2. Morlet N, Daniell M. Microbial keratitis: what's the preferred initial therapy? View 2: Empirical fluoroquinolone therapy is sufficient initial treatment. $\mathrm{Br} \mathrm{J}$ Ophthalmol. 2003;87:1169-72.

3. Hau SC, Dart JK, Vesaluoma M, Parmar DN, Claerhout I, Bibi K, et al. Diagnostic accuracy of microbial keratitis with in vivo scanning laser confocal microscopy. $\mathrm{Br}$ J Ophthalmol. 2010;94:982-7.

4. Chidambaram JD, Prajna NV, Larke NL, Palepu S, Lanjewar S, Shah M, et al. Prospective study of the diagnostic accuracy of the in vivo laser scanning confocal microscope for severe microbial keratitis. Ophthalmology. 2016;123:2285-93.

5. Chidambaram JD, Prajna NV, Larke N, Macleod D, Srikanthi P, Lanjewar S, et al. In vivo confocal microscopy appearance of Fusarium and Aspergillus species in fungal keratitis. Br J Ophthalmol. 2017;101:1119-23.

6. Kanavi MR, Javadi M, Yazdani S, Mirdehghanm S. Sensitivity and specificity of confocal scan in the diagnosis of infectious keratitis. Cornea. 2007;26:782-6.

7. Vaddavalli PK, Garg P, Sharma S, Sangwan VS, Rao GN, Thomas R. Role of confocal microscopy in the diagnosis of fungal and acanthamoeba keratitis. Ophthalmology. 2011;118:29-35.

8. Goh JWY, Harrison R, Hau S, Alexander CL, Tole DM, Avadhanam VS. Comparison of in vivo confocal microscopy, PCR and culture of corneal scrapes in the diagnosis of acanthamoeba keratitis. Cornea. 2018;37:480-5. 
9. Alomar T, Matthew M, Donald F, Maharajan S, Dua HS. In vivo confocal microscopy in the diagnosis and management of acanthamoeba keratitis showing new cystic forms. Clin Exp Ophthalmol. 2009;37:737-9.

10. Vaddavalli PK, Garg P, Sharma S, Thomas R, Rao GN. Confocal microscopy for Nocardia keratitis. Ophthalmology. 2006;113:1645-50.

11. Hsu HY, Ernst B, Schmidt EJ, Parihar R, Horwood C, Edelstein SL. Laboratory results, epidemiologic features, and outcome analyses of microbial keratitis: a 15year review from St. Louis. Am J Ophthalmol. 2019;198:54-62.

12. Sand D, She R, Shulman IA, Chen DS, Schur M, Hsu HY. Microbial keratitis in los angeles: the doheny eye institute and the los angeles county hospital experience. Ophthalmology. 2015;122:918-24.

13. Lichtinger A, Yeung SN, Kim P, Amiran MD, lovieno A, Elbaz $U$, et al. Shifting trends in bacterial keratitis in Toronto: an 11-year review. Ophthalmology. 2012;119:1785-90.

14. Leck AK, Thomas PA, Hagan M, Kaliamurthy J, Ackuaku E, John M, et al. Aetiology of suppurative corneal ulcers in Ghana and south India, and epidemiology of fungal keratitis. Br J Ophthalmol. 2002;86:1211-5.

15. Truong DT, Bui M-T, Cavanagh HD. Epidemiology and outcome of microbial keratitis: private university versus urban public hospital care. Eye Contact lens. 2018;44:S82-S86.

16. Morlet N, Minassian D, Butcher J. Risk factors for treatment outcome of suspected microbial keratitis. Ofloxacin Study Group. Br J Ophthalmol. 1999;83:1027-31.

17. Carnt N, Hoffman JJ, Verma S, Hau S, Radford CF, Minassian DC, et al. Acanthamoeba keratitis: confirmation of the UK outbreak and a prospective case-control study identifying contributing risk factors. Br J Ophthalmol. 2018;102:1621-8.

18. lovieno A, Gore DM, Carnt N, Dart JK. Acanthamoeba sclerokeratitis: epidemiology, clinical features, and treatment outcomes. Ophthalmology. 2014;121:2340-7.

19. Lehmann OJ, Green SM, Morlet N, Kilvington S, Keys MF, Matheson MM, et al. Polymerase chain reaction analysis of corneal epithelial and tear samples in the diagnosis of Acanthamoeba keratitis. Investig Ophthalmol Vis Sci. 1998;39:1261-5.

20. Kuo MT, Chang HC, Cheng CK, Chien CC, Fang PC, Chang TC. A highly sensitive method for molecular diagnosis of fungal keratitis: a dot hybridization assay. Ophthalmology. 2012;119:2434-42.

21. Tan SZ, Walkden A, Au L, Fullwood C, Hamilton A, Qamruddin A, et al. Twelve-year analysis of microbial keratitis trends at a UK tertiary hospital. Eye. 2017;31:1229-36.

22. Kim E, Chidambaram JD, Srinivasan M, Lalitha P, Wee D, Lietman TM, et al. Prospective comparison of microbial culture and polymerase chain reaction in the diagnosis of corneal ulcer. Am J Ophthalmol. 2008;146:714-23. 723 e711.

23. Eleinen KG, Mohalhal AA, Elmekawy HE, Abdulbaki AM, Sherif AM, El-Sherif RH, et al. Polymerase chain reaction-guided diagnosis of infective keratitis-a hospital-based study. Curr Eye Res. 2012;37:1005-11.

24. Panda A, Pal Singh T, Satpathy G, Wadhwani M, Monika M. Comparison of polymerase chain reaction and standard microbiological techniques in presumed bacterial corneal ulcers. Int Ophthalmol. 2015;35:159-65.

25. Harris KA, Hartley JC. Development of broad-range 16S rDNA PCR for use in the routine diagnostic clinical microbiology service. J Med Microbiol. 2003;52:685-91.

26. Tananuvat N, Salakthuantee K, Vanittanakom N, Pongpom M, Ausayakhun S. Prospective comparison between conventional microbial work-up vs PCR in the diagnosis of fungal keratitis. Eye. 2012;26:1337-43.

27. Vengayil S, Panda A, Satpathy G, Nayak N, Ghose S, Patanaik D, et al. Polymerase chain reaction-guided diagnosis of mycotic keratitis: a prospective evaluation of its efficacy and limitations. Invest Ophthalmol Vis Sci. 2009;50:152-6.

28. Ferrer C, Alio JL. Evaluation of molecular diagnosis in fungal keratitis. ten years of experience. J Ophthalmic Inflamm Infect. 2011;1:15-22.

29. Boggild AK, Martin DS, Lee TY, Yu B, Low DE. Laboratory diagnosis of amoebic keratitis: comparison of four diagnostic methods for different types of clinical specimens. J Clin Microbiol. 2009;47:1314-8.

30. Kowalski RP, Melan MA, Karenchak LM, Mammen A. Comparison of validated polymerase chain reaction and culture isolation for the routine detection of acanthamoeba from ocular samples. Eye Contact Lens. 2015;41:341-3.

31. Pasricha G, Sharma S, Garg P, Aggarwal RK. Use of 18 S rRNA gene-based PCR assay for diagnosis of acanthamoeba keratitis in non-contact lens wearers in India. J Clin Microbiol. 2003;41:3206-11.

32. Tu EY, Joslin CE, Sugar J, Booton GC, Shoff ME, Fuerst PA. The relative value of confocal microscopy and superficial corneal scrapings in the diagnosis of Acanthamoeba keratitis 1. Cornea. 2008;27:764-72.
33. De Craene S, Knoeri J, Georgeon C, Kestelyn P, Borderie VM. Assessment of confocal microscopy for the diagnosis of polymerase chain reaction-positive acanthamoeba keratitis: a case-control study. Ophthalmology. 2018;125:161-8.

34. Carnt N, Hoffman J, Verma S, Hau S, Radford CF, Minassian DC, et al. Acanthamoeba keratitis: confirmation of the UK outbreak and a prospective case-control study identifying contributing risk factors. Br J Ophthalmol. 2018;102:1621-8.

\section{AUTHOR CONTRIBUTIONS}

$\mathrm{JH}$ and JD performed the literature search. JH, JD and $\mathrm{SH}$ were responsible for the study conception and design. $\mathrm{JH}, \mathrm{NC}, \mathrm{GC}$ and $\mathrm{SH}$ collected the data. $\mathrm{JH}$ and $\mathrm{SH}$ performed the statistical analysis. $\mathrm{JH}, \mathrm{SH}, \mathrm{SD}$ and JD were responsible for the interpretation of the data. JH drafted the manuscript. All authors critically revised the manuscript for important intellectual content. JH, SH and JD accessed and verified the data. SH and JD were the study supervisors. All authors had full access to all the data in the study and had final responsibility for the decision to submit for publication.

\section{FUNDING}

$\mathrm{JHH}$ is supported by Wellcome Trust (207472/Z/17/Z) and an Academic Clinical Fellowship from the National Institute for Health Research (NIHR, 3071). Part of John Dart's salary was paid by the NIHR Biomedical Research Centre (BRC) at Moorfields Eye Hospital and the UCL Institute of Ophthalmology The funders had no role in study design, data collection and analysis, decision to publish, or preparation of the manuscript.

\section{COMPETING INTERESTS}

The authors declare no competing interests.

\section{ADDITIONAL INFORMATION}

Supplementary information The online version contains supplementary material available at https://doi.org/10.1038/s41433-021-01812-7.

Correspondence and requests for materials should be addressed to Jeremy J. Hoffman.

Reprints and permission information is available at http://www.nature.com/ reprints

Publisher's note Springer Nature remains neutral with regard to jurisdictional claims in published maps and institutional affiliations.

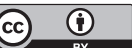

Open Access This article is licensed under a Creative Commons Attribution 4.0 International License, which permits use, sharing, adaptation, distribution and reproduction in any medium or format, as long as you give appropriate credit to the original author(s) and the source, provide a link to the Creative Commons license, and indicate if changes were made. The images or other third party material in this article are included in the article's Creative Commons license, unless indicated otherwise in a credit line to the material. If material is not included in the article's Creative Commons license and your intended use is not permitted by statutory regulation or exceeds the permitted use, you will need to obtain permission directly from the copyright holder. To view a copy of this license, visit http://creativecommons. org/licenses/by/4.0/.

(c) The Author(s) 2021 